\title{
Interdependenzen zwischen Raumordnung und Umwelt - Eine Übersicht über aktuelle Integrationsansätze in Theorie und Praxis
}

\section{Einleitung}

«Ökologische Planung», «ökologische Regionalentwicklung», «regionalisierte Umweltpolitik»... solche und ähnliche Stichworte sind in der wissenschaftlichen Literatur immer häufiger anzutreffen. Ökologie, Raumplanung und Regionalwissenschaft treten offensichtlich in immer engeren Kontakt zueinander. Dabei ist nicht nur - entsprechend der «Ökologisierung» sämtlicher Lebensbereiche - ein Eindringen der Ökologie in Fragestellungen der Raumordnung festzustellen. Auch die Ökologie wird zunehmend hinsichtlich räumlicher Aspekte und Dimensionen analysiert. Die wissenschaftliche Auseinandersetzung mit dem Grenzbereich zwischen Umwelt und Raumordnung hat mittlerweile Dimensionen erreicht, welche auch dem eingeweihten Beobachter die Orientierung erschweren.

Hier kann und soll nicht auf eine Diskussion über das Wesen der Geographie eingetreten werden, doch können zweifellos Raumordnung und Ökologie - zumindest in dieser allgemeinen Form - als zwei der wichtigsten Fragestellungen in der Geographie bezeichnet werden (BAHRENBERG 1983, 63; LESER 1980, 43f.). Wenn Raumordnung und Umwelt zunehmend ineinandergreifen, so ist dies sicherlich auch für die Geographie von Bedeutung. Der Zweck des vorliegenden Beitrages besteht darin, eine Übersicht über aktuelle Integrationsansätze zu geben, diese zu ordnen und zu gliedern.

Bevor allerdings auf eine inhaltliche Diskussion eingetreten werden kann, sind die Begriffe «Raumordnung» und «Umwelt» bzw. «Ökologie» zu klären. Für die Definition von Raumordnung halten wir uns an LENDI/ELSASSER $(1985,11)$ : «Raumordnung ist der gegebene oder erstrebenswerte künftige Zustand eines Gebietes. Die Raumordnung kann auch als Tätigkeit verstanden werden.» Raumordnung ist als Oberbegriff für die Sachbereiche Raumplanung sowie Regionalentwicklung und Regionalpolitik zu verstehen (LENDI/ELSASSER 1985, 133). Umwelt bzw. Ökologie werden entsprechend den unterschiedlichen Konzeptionen von verschiedenen Disziplinen und Autoren sehr weit gefaßt. Es können Wirkungsmechanismen von Ökosystemen, Umwelt- oder Naturraumpotentiale, Umweltprobleme, Umweltschutzmaßnahmen usw. angesprochen sein. Dies mag zwar als unbefriedigend unpräzis erscheinen, widerspiegelt aber die uneinheitliche Begriffsverwendung in den nachfolgend diskutierten Integrationsbereichen zur Raumordnung.

Die im Grenzbereich zwischen Raumordnung und Umwelt angesiedelten Ansätze und Untersuchungen lassen sich u. E. in vier Gruppen untergliedern*: raumwirtschaftliche Aspekte der Umweltnutzung, raumwirtschaftliche Aspekte der Umweltpolitik, ökologische Aspekte der Regionalpolitik und die ökologische Dimension in der Raumplanung. In Tabelle 1 haben wir die in diesem Beitrag behandelten Aspekte stichwortartig festgehalten. Die in Klammern angegebenen Zahlen bezeichnen die Abschnitte, in denen die Fragenkreise zur Sprache kommen.

\section{Raumwirtschaftliche Aspekte der Umweltnutzung}

Unter dem Titel «Raumwirtschaftliche Aspekte der Umweltnutzung» fassen wir verschiedene Ansätze zusammen, welche die Umweltnutzung in räumlicher Hinsicht analysieren oder Umweltaspekte in die Analyse regionaler Entwicklungsprozesse einbeziehen. In diesem Abschnitt ist von der «reinen» Umweltnutzung die Rede, d. h. es wird vorerst von staatlichen umweltpolitischen Eingriffen abgesehen. Die raumwirtschaftlichen Aspekte der Umweltnutzung können ihrerseits in drei größere Bereiche untergliedert werden:

(1) Die raumwirtschaftliche Bedeutung der Umweltausstattung einer Region

(2) Die räumliche Dimension der Umweltproblematik

(3) Die raumwirtschaftliche Bedeutung von Umweltexternalitäten.

*Einige wichtige Anregungen erhielt der Autor im Seminar zur Regionalwirtschaft von Prof. A. Rossi "Regionale Betrachtung in der Umweltökonomie», Universität Zürich, Sommersemester 1988.

Daniel Wachter, dipl. Geograph, Geograph. Institut der Universität Zürich-Irchel, Winterthurerstr. 190, 8057 Zürich 
Tab. 1: Interdependenzen zwischen Raumordnung und Umwelt: tabellarische Übersicht über die behandelten Aspekte

\begin{tabular}{|c|c|c|c|}
\hline $\begin{array}{l}\text { Raumwirtschaft- } \\
\text { liche Aspekte der } \\
\text { Umweltnutzung }\end{array}$ & $\begin{array}{l}\text { Raumwirtschaft- } \\
\text { liche Aspekte der } \\
\text { Umweltpolitik }\end{array}$ & $\begin{array}{l}\text { Ökologische Aspekte } \\
\text { der Regionalpolitik }\end{array}$ & $\begin{array}{l}\text { Die ökologische } \\
\text { Dimension in der } \\
\text { Raumplanung }\end{array}$ \\
\hline $\begin{array}{l}\text { Die raumwirtschaft- } \\
\text { iiche Bedeutung der } \\
\text { Umweltausstattung } \\
\text { einer Region (2.1.) }\end{array}$ & $\begin{array}{l}\text { Räumliche Vertei- } \\
\text { lungsaspekte der } \\
\text { Umweltpolitik (3.1.) }\end{array}$ & $\begin{array}{l}\text { Traditionelle Re- } \\
\text { gionalpolitik und } \\
\text { Umweltschutz (4.1) }\end{array}$ & (Kapitel 5.) \\
\hline $\begin{array}{l}\text { Die räumliche Di- } \\
\text { mension der Um- } \\
\text { weltproblematik } \\
(2.2 .) \\
\text { Die raumwirt- } \\
\text { schaftliche Be- } \\
\text { deutung von } \\
\text { Umweltexter- } \\
\text { nalitäten (2.3.) }\end{array}$ & $\begin{array}{l}\text { Folgerungen für } \\
\text { eine effizienzori- } \\
\text { entierte Umwelt- } \\
\text { politik aufgrund } \\
\text { der räumlichen } \\
\text { Dimension der } \\
\text { Umweltproble- } \\
\text { matik: regiona- } \\
\text { lisierte Umwelt- } \\
\text { politik (3.2.) }\end{array}$ & $\begin{array}{l}\text { Neuere regional- } \\
\text { politische Entwürfe } \\
\text { und Umweltschutz } \\
\text { (4.2.) } \\
\text { Umweltschutz } \\
\text { als Mittel der Re- } \\
\text { gionalpolitik (4.3.) }\end{array}$ & \\
\hline
\end{tabular}

\subsection{Die raumwirtschaftliche Bedeutung der Umweltausstattung einer Region}

Hierbei handelt es sich um einen teilweise sehr traditionellen Ansatz, welcher den Zusammenhang zwischen Wirtschaftsstruktur, Wohlstand und Ausstattung einer Region mit Umweltgütern untersucht. Diese Thematik wurde bereits von den frühen Theorien der industriellen Standortwahl aufgenommen. In der Industriestandorttheorie von Alfred Weber beispielsweise (vgl. dazu SCHÄTZL 1981, 31f.) beeinflussen drei Standortfaktoren die industrielle Standortwahl: Transportkosten, Arbeitskosten und Agglomerationsvorteile. Die Transportkosten nehmen in Webers Theorie eine zentrale Stellung ein, wobei sie nur vom Gewicht der transportierten Materialien abhängen. Aufgrund der spezifischen Annahmen üben Rohstoffvorkommen einen großen Einfluß auf die industrielle Standortwahl aus.

Die Theorie der industriellen Standortwahl wurde von verschiedenen Autoren weiterentwickelt, welche die übrigen Kostenkomponenten neben den Transportkosten stärker sowie auch die Ertragsseite berücksichtigten. Neuere Arbeiten (z. B. LLOYD/DIKKEN 1977) zeigen klar, daß die Transportkosten für die industrielle Standortwahl wegen des technischen Fortschritts im Verkehrssektor kaum noch von Bedeutung sind, andere Kostenkomponenten sowie Ertragsfaktoren somit wichtiger werden, was im allgemeinen bedeutet, daß der Standortfaktor «natürliche Ressourcen» abgewertet wird. Als Beispiel mag hier der Hinweis auf das Standortwahlverhalten der Stahlindustrie, welche sich im Laufe der Zeit immer weiter von den Rohstoffvorkommen wegbewegte, genügen. Es wäre jedoch voreilig, aufgrund der obigen Argumente die raumwirtschaftliche Bedeutung der Umweltausstattung einer Region als generell nur mehr gering einzustufen. In bezug auf die Schweiz wies BRUGGer $(1985,211-215)$ auf die folgenden raumwirtschaftlich bedeutenden Umweltfaktoren hin:

\section{Landschaft}

Die Entwicklung des schweizerischen Berggebietes, eines ehemals sehr wirtschaftsschwachen Teilraumes der Schweiz, beruht zum großen Teil auf der Inwertsetzung der Ressource «Landschaft». Die Landschaft stellt das «ursprüngliche Angebot» des Tourismus dar (KRIPPENDORF u. a. 1987, 84) und ist «Rohstoff, Existenzgrundlage und Wirtschaftsmotor des Tourismus» (KRIPPENDORF 1984, 603). 
Kulturland und Wald

In weiten Teilen des ländlichen Raumes der Schweiz stellen auch heute noch die Land- und die Forstwirtschaft, welche direkt Naturraumpotentiale nutzen, wichtige wirtschaftliche Stützen dar.

\section{Boden als Baugrund}

In diesem Zusammenhang besonders wichtig ist die Rolle des Bodenpreises. Das Bodenpreisgefälle von den Zentren in die Peripherien entmischt die verschiedenen Raumnutzungen. Das Bodenpreisgefälle ist allerdings nur besonders stark zwischen City und Agglomerationsumland, während es dann in einem weiteren räumlichen Umland deutlich abnimmt. Der Bodenfaktor ist deshalb vor allem kleinräumig, innerhalb von Agglomerationsgebieten von raumwirtschaftlichem Einfluß.

\section{Reliefenergie der Topographie}

Dieses Naturraumpotential des Berggebietes wurde in der Vergangenheit systematisch zur Elektrizitätsgewinnung genutzt. In vielen Bergkantonen stellt die Elektrizitätswirtschaft neben dem Tourismus die zweite tragende Säule der Volkswirtschaft dar, indem sie - häufig in sehr abgelegenen Gebieten Einkommen und Beschäftigung schafft.

Aus diesen Bemerkungen kann gefolgert werden, daß das Umweltpotential zwar für die industrielle Standortwahl kaum noch oder nur in Einzelfällen wichtig ist, daß es aber auf anderen Gebieten (z. B. Tourismus) sehr wohl von raumwirtschaftlicher Bedeutung ist, und dies insbesondere für traditionell oder ehemals wirtschaftsschwache Regionen.

\subsection{Die räumliche Dimension der Umweltproblematik}

Einen zweiten raumwirtschaftlichen Aspekt der Umweltnutzung bildet der Umstand, daß Umweltprobleme und die zugrunde liegenden Parameter nicht über ein ganzes Land uniform verteilt sind. Als wichtige Einflußgrößen auf die regionale Umweltsituation können angeführt werden (vgl. dazu

z. B. BENKERT 1981, 42f., SIEBERT u. a. 1979, 3):

- In der Region erzeugte Emissionen und sonstige Belastungen

- Assimilationskapazität des regionalen Ökosystems

- In der Region anfallende Immissionen

- Anzahl und Art der beeinträchtigungsempfindlichen Nutzungen und Aktivitäten

- Bewertung der Umweltqualität.

$\mathrm{Da}$ die regionale Umweltsituation durch den Umfang an belastenden Aktivitäten bestimmt wird, ist offensichtlich. Die ökologische Kapazität bestimmt, ob ein gegebenes Emissionsniveau zu Immissionen führt. Nur die über die ökologische Kapazität hinausgehenden Emissionen können belastend auf andere Nutzungsarten wirken (BENKERT 1981, 61). Sodann hängt der Schaden von Immissionen davon ab, ob und welche empfindlichen Aktivitäten betroffen sind. Ob schließlich eine physische Beeinträchtigung von den Menschen als Schaden empfunden wird, hängt nicht zuletzt auch von Präferenzen und Wertungen ab. Um die räumliche Dimension der Umweltproblematik an einem Beispiel zu verdeutlichen, sei die besondere Situation der Luftverschmutzung in Großstätten erwähnt.

Die Thematisierung der räumlichen Dimension der Umweltproblematik ist relevant für das Auffinden zweckmäßiger Lösungsstrategien. Eine national uniforme Umweltpolitik ist damit zu hinterfragen (vgl. Kap. 3.2).

\subsection{Die raumwirtschaftliche Bedeutung von Umweltexternalitäten}

Den dritten raumwirtschaftlichen Aspekt der Umweltnutzung bilden die externen Effekte im Umweltbereich oder Umweltexternalitäten. Eine Sonderform stellen die sogenannten Spillovers dar. Externe Effekte sind in der Wirtschaftstheorie als gegenseitige Einwirkungen von Wirtschaftssubjekten definiert, die vom Markt nicht erfaßt werden. Kosten, die vom Verursacher auf Drittpersonen abgewälzt werden, werden als negative externe Effekte bezeichnet. Positive externe Effekte liegen vor, wenn jemand Nutzen oder Erträge für Dritte erbringt, ohne dafür bezahlt zu werden. Spillovers sind räumliche externe Effekte, d. h. positive und negative Auswirkungen einer Region auf andere Regionen, für welche keine Abgeltung bezahlt wird. Externe Effekte und Spillovers im Umweltbereich können sowohl materieller (unabgegoltene Übertragung von physischen Stoffen) als auch immaterieller Art sein (Umweltdienste). Sie werden in der regionalwissenschaftlichen Literatur am häufigsten in den folgenden Ausprägungen aufgegriffen:

a) Agglomerationsnachteile

b) Schadstoffspillovers

c) positive externe Effekte des ländlichen Raumes

\section{a) Agglomerationsnachteile}

Die das Stadtwachstum bestimmenden Agglomerationsvor- und -nachteile sind meist externe Effekte. Regionale Polarisationsprozesse können teilweise damit erklärt werden. In der ökonomischen Theorie der optimalen Stadtgröße (vgl. dazu z. B. RICHARDson 1978, 73f.) wird die Agglomerationsgröße mit dem Verhältnis von Kosten und Nutzen aus der Zusammenballung erklärt. Hier steht nicht der Raum zur Verfügung, um die ökonomische Argumentation ausführlich wiederzugeben; wir müssen uns auf eine abgekürzte Darstellung beschränken. Die Existenz von negativen Umweltexternalitäten (z. B. Luftverschmutzung, Verkehrsstaus) bedeutet, daß die in der Stadt ansässigen Wirtschaftssubjekte nicht die vollen sozialen Kosten ihrer Handlungen tragen. Die Agglomerationsnachteile werden zu ge- 
ring ausgewiesen, wodurch die Stadt über die volkswirtschaftlich optimale Größe hinauswächst.

\section{b) Schadstoffspillovers}

Die raumwirtschaftliche Bedeutung von Schadstoffspillovers beschreibt BENKERT $(1981,113)$ wie folgt: «Im Vergleich zweier ökologisch interdependenter Raumpunkte bedeutet das Wirken der Diffusionsfähigkeit allgemein, daß „externe” Assimilationskapazität mitgenutzt wird, ohne daß der Verursacher dieser Belastungen die ökologischen Kosten zu tragen hat. Für den betroffenen Raumpunkt verringert sich zugleich das verfügbare ökologische Potential. Die Erhöhung des Emissionsniveaus in einer Region bewirkt also ökonomische und ökologische Interdependenzen mit anderen Regionen, die die relative Wohlfahrtsposition der beteiligten Regionen dadurch in spezifischer Weise verändern, daß die auftretenden ökologischen Kosten intersektoral wie interregional von den Verursachern des erhöhten Emissionsniveaus abgewälzt werden können.» Die Relevanz dieser theoretischen Erörterungen ist für die Schweiz z. B. dadurch gegeben, daß die überwiegend aus den Ballungsräumen stammende Luftverschmutzung heute als eine wesentliche Ursache für den Vitalitätsverlust des Waldes allgemein und der Bergwälder im speziellen betrachtet wird.

c) positive externe Effekte des ländlichen Raumes

Neben den negativen Umweltexternalitäten sind auch positive Umweltexternalitäten, welche vorwiegend dem ländlichen Raum zuzuordnen sind, von raumwirtschaftlicher Bedeutung. Mit HOLLENSTEIN/LOERTSCHER $(1980,50)$ kann die Problematik wie folgt dargelegt werden: "Im Rahmen einer weitergehenden Interpretation regionaler Externalitäten lassen sich zusätzliche Ausgleichszahlungen an einkommensschwache Regionen begründen: Zwischen Agglomerationen und Peripherie bestehen notwendigerweise Komplementaritäten, die sich in interregionalen Unterschieden hinsichtlich der Wirtschaftsstruktur und damit der Wirtschaftskraft niederschlagen; eine Zentrumsregion ohne zugehöriges ... Hinterland ist kaum denkbar. In dieser Sicht dienen Ausgleichszahlungen dazu, die für Randregionen mit der Erfüllung von Komplementärfunktionen per Saldo anfallenden wirtschaftlichen Nachteile abzugelten.»

\section{Raumwirtschaftliche Aspekte der Umweltpolitik}

Einen zweiten großen Problemkreis im Grenzbereich zwischen Raumordnung und Umwelt bilden raumwirtschaftliche Aspekte der Umweltpolitik. In dieser Hinsicht sind zwei Fragenkreise zu unterscheiden. Einerseits werden räumliche Verteilungsaspekte umweltpolitischer Strategien und Maßnah- men, andererseits Effizienzaspekte der Umweltpolitik diskutiert, welche sich aufgrund der räumlichen Dimension der Umweltproblematik ergeben. Allerdings bestehen vielfache Überschneidungen zwischen Verteilungs- und Effizienzaspekten.

\subsection{Räumliche Verteilungsaspekte der Umweltpolitik}

Verteilungswirkungen umweltpolitischer Maßnahmen sind sehr komplex. In einer vollständigen Analyse müßten die Verteilung sowohl der Umweltschutzkosten als auch der Umweltverbesserungen, sowohl Primär- als auch Folgeeffekte berücksichtigt werden (vgl. dazu z. B. ZIMMERMANN 1985). Wir müssen uns hier auf einige allgemeine Bemerkungen beschränken.

Ganz generell gilt, daß eine strikte auf umweltpolitische Effizienz ausgerichtete Umweltpolitik dann keine Konflikte mit regionalpolitischen Zielsetzungen erzeugt, wenn die regionalpolitischen Problemregionen durch eine hohe Umweltqualität und niedrige Umweltschutzkosten gekennzeichnet sind, die wirtschaftsstarken Regionen andererseits hohe Umweltbelastung und Umweltschutzkosten aufweisen (R. L. FREY 1979a, 101). Ernsthafte Konflikte treten auf, wenn die Problemregionen gleichzeitig gravierende, die reichen Regionen aber relativ geringe Umweltprobleme aufweisen. Dies trifft beispielsweise auf gewisse altindustrielle Regionen $\mathrm{zu}$ (Schwerindustrieregionen). Aber auch ländlich-periphere Regionen können zumindest teilweise von diesem Sachverhalt betroffen sein. In bezug auf den Verkehr gilt, daß in ländlich-peripheren Regionen wegen der dispersen Besiedlung hohe durchschnittliche Fahrtdistanzen bestehen und privater Motorfahrzeugverkehr kaum durch öffentlichen Verkehr ersetzt werden kann. Deshalb würden diese Regionen von umweltpolitischen Maßnahmen im Verkehrsbereich, beispielsweise einer Emissionssteuer, stärker betroffen als städtische Räume. Der Umweltpolitik bieten sich in dieser Situation nach R. L. FREY (1979a, 101) drei Möglichkeiten:

- Tolerierung der disparitätenfördernden Effekte,

- Kompensation der benachteiligten Regionen z. B. über einen Finanzausgleich,

- umweltpolitische Kompromisse gegenüber den regionalpolitischen Problemregionen.

Die raumwirtschaftlichen Effekte hängen allerdings nicht nur von der interregional unterschiedlichen Umweltproblematik ab, sondern auch von den angewandten Instrumenten. R. L. FREY u. a. (1985, 115-118) gehen davon aus, daß marktwirtschaftliche Instrumente (z. B. Emissionssteuern, Energiesteuern usw.) mit negativen Verteilungswirkungen für ärmere Regionen und Branchen mit Strukturproblemen verbunden sind, währenddem direkte Kontrollen (Ge-, Verbote) und Maßnahmen nach dem Gemeinlastprinzip (Subventionen für Umwelt- 
schutzmaßnahmen) positive Verteilungswirkungen für die angesprochenen Regionen aufweisen. Sie kommen zum Schluß, «daß im großen und ganzen diejenigen Instrumente politisch wenig attraktiv sind, die in wirtschaftlicher Hinsicht gut abschneiden. Dies hängt ... damit zusammen, daß die effizienten Instrumente tendenziell unerwünschte Verteilungswirkungen haben. Es handelt sich dabei einmal mehr um den altbekannten Konflikt zwischen Effizienz und Verteilungsgerechtigkeit» (S. 116).

\subsection{Effizienzaspekte}

Im letzten Abschnitt diskutierten wir räumliche Verteilungsaspekte national einheitlicher Umweltpolitik. In diesem Abschnitt nun gehen wir auf die Frage ein, welche Folgerungen sich für die Umweltpolitik im Hinblick auf das Erreichen von umweltpolitischer Effizienz aufgrund der räumlichen Dimension der Umweltproblematik ergeben. Die nachfolgenden Überlegungen kreisen um den Begriff der regionalisierten Umweltpolitik.

Die in Abschnitt 2.2 dargelegten Parameter der regionalen Umweltsituation, auf welche uniforme nationale Umweltpolitiker bisher kaum Rücksicht genommen haben, veranlassen SIEBERT $(1979,3)$, eine regionalisierte Umweltpolitik zu fordern: «The criteria of economic efficiency ... clearly dictate a regional differentiation of environmental policy instruments for the following reasons ... Environmental quality may be more scarce in one region relative to another area because: (i) the region has a smaller assimilative capacity; (ii) consumers value environmental quality more highly and can manifest their preference in the political process accordingly; (iii) the region is more densely populated and a given quantity of pollutants creates greater social damage; or (iv) there is a larger quantity of emissions because of industrial structure, production, and abatement technology. As a result of these factors, we also can expect, that the target values of environmental quality will differ among regions.»

ZIMMERMANN/NIJKAMP $(1986,38)$ unterscheiden hinsichtlich der Ausgestaltung regionalisierter Umweltpolitik die Regionalisierung von Instrumenten bzw. deren Dosierung und die Regionalisierung von Zielen der Umweltqualität und leiten daraus drei Hauptausprägungen regionalisierter Umweltpolitik ab:

a) Umweltpolitik auf nationaler Entscheidungsebene; Regionalisierung von Emissionsnormen; identische Immissionsnormen

b) Umweltpolitik auf nationaler Entscheidungsebene; Regionalisierung von Immissionsnormen (Politik der Vorranggebiete: Vorranggebiete für umweltbelastende Aktivitäten, für Naturschutz usw.)

c) Regionalisierte Umweltpolitik auf regionaler Entscheidungsebene (Regionalisierung sowohl von Emissions- als auch von Immissionsnormen).

Welche dieser drei Strategien vorteilhaft ist, ist von den jeweiligen Umweltproblemen und weiteren Kriterien abhängig.

Effizienzorientierte regionalisierte Umweltpolitik beeinflußt ihrerseits - damit knüpfen wir an Abschnitt 3.1 an - die Raumstruktur. Welche raumwirtschaftlichen Auswirkungen sind von regionalisierter Umweltpolitik zu erwarten? In bezug auf diese Frage möchten wir zunächst das neoklassisch geprägte «räumliche Allokationsmodell» von SIEBERT (1978, 123f.) vorstellen. Auf der Basis der üblichen restriktiven Annahmen neoklassischer raumwirtschaftlicher Modelle postuliert er für einen ZweiRegionen-Fall, bei dem die beiden Regionen vorerst als identisch angenommen werden, daß sich eine auftretende Abweichung in der Umweltbelastung in interregional unterschiedlichen Emissionssteuersätzen niederschlagen müsse. Die Differenz der Emissionssteuersätze löse schließlich interregionale Produktionsfaktorwanderungen von der relativ stärker belasteten in die relativ schwächer belastete Region aus und führe zu einer Angleichung des Emissionssteuersatzes. Grundsätzlich geht sIEBERT davon aus, daß regionalisierte Umweltpolitik räumlich ausgleichend wirke. "Insbesondere ist zu erwarten, daß die bisher falsch ausgewiesenen Agglomerationsvorteile durch die korrekte Ausweisung eines Teils der sozialen Kosten der Agglomeration auf ein realistischeres Maß reduziert werden» (SIEBERT 1978, 138).

Hier kann nicht eine ausführliche Kritik an diesem neoklassischen Modell und an der räumlichen Ausgleichsthese vorgenommen werden. Es ist jedoch zu betonen, daß sich sIEBERTS Ausgleichsthese in erster Linie auf den Emissionssteuersatz bezieht. SIEBERT selbst sagt, daß z. B. interregionale Unterschiede in der Umweltausstattung (Assimilationskapazität) oder interregionale Unterschiede in der Bewertung von Umweltqualität zu Spezialisierungen der Teilräume auf gewisse Raumfunktionen oder Produktionszweige und eventuell $\mathrm{zu}$ unterschiedlichen Wohlfahrtsniveaus führen können.

\section{4. Ökologische Aspekte der Regionalpolitik}

Regionalpolitik war in der Vergangenheit vor allem regionale Wirtschaftspolitik (R. L. FREY 1985, 5), deren Hauptziel der regionale wirtschaftliche Ausgleich war. In neueren Publikationen werden aber vermehrt die Vielgestaltigkeit der Regionalproblematik sowie weitere, außerökonomische Zielsetzungen der Regionalpolitik betont. BRUGGER/FREY $(1985,38 f$.) nahmen u. a. aufgrund der räumlichen Dimension der Umweltproblematik und wegen des Spilloverproblems den Landschafts- und Umwelt- 
schutz sogar in den regionalpolitischen Zielkatalog auf. Es liegt allerdings auf der Hand, daß die Zielerreichung u. a. von der Gewichtung abhängt. In diesem Kapitel analysieren wir verschiedene regionalpolitische Strategien in bezug auf ihre umweltbezogenen Implikationen. Im ersten Abschnitt gehen wir auf zwei traditionelle Strategien ein. Im zweiten Abschnitt werden einige neuere Entwürfe kurz angesprochen, welche explizit oder implizit die Umwelt berücksichtigen. Im letzten Abschnitt werden einige neuere Ansätze vorgestellt, welche Umweltschutz als Mittel der Regionalpolitik einsetzen.

\subsection{Traditionelle Regionalpolitik und Umweltschutz}

Als traditionelle Regionalpolitik bezeichnen wir Strategien, welche am Anfang der offiziellen staatlichen Regionalpolitik standen, in den sechziger und siebziger Jahren entworfen wurden und vielfach heute noch die Basis der Regionalpolitik darstellen. Es handelt sich um die Strategien Infrastrukturausbau und (einzelbetriebliche) Wirtschaftsförderung. Während mit ersterer indirekt durch Aufwertung regionaler Standortfaktoren die Schaffung von Arbeitsplätzen gefördert werden soll, wird die Arbeitsplatzschaffung bei letzterer direkt durch Darlehen, Bürgschaften, Zinskostenbeiträge usw. an Unternehmen unterstützt. Beide Strategien weisen keinen direkten Umweltbezug auf. Ursprünglich waren beide auf regionalen Ausgleich und quantitatives Wachstum in den Förderregionen ausgerichtet. Erst später wurden dann vermehrt qualitative Aspekte berücksichtigt: bei der einzelbetrieblichen Wirtschaftsförderung z. B. der Innovationsgehalt der geförderten Projekte, die Qualität der Arbeitsplätze und die Umweltverträglichkeit. Nach zIMMERMANN/NIJKAMP $(1986,57)$ ist aber davon auszugehen, daß zwischen traditionellen regionalpolitischen Strategien und Umweltpolitik wesentliche Zielkonflikte bestehen.

\subsection{Neuere regionalpolitische Entwürfe und Umweltschutz}

In diesem Abschnitt sollen einige neuere regionalpolitische Strategien angeschnitten werden, die wegen der Betonung qualitativer Aspekte der Regionalentwicklung zumindest potentiell umweltverträgliche Strategien sind. Für die Auswahl lehnen wir uns an ZIMMERMANN/NIJKAMP (1986, 67f.) an und gehen - aus Platzgründen nur kurz antippend - auf die Strategien Regionalismus, endogene Regionalpolitik und innovationsorientierte Regionalpolitik ein.

Regionalismus ist nach ZIMMERMANN/NIJKAMP vor allem im Zusammenhang mit dem Wertewandel in der Gesellschaft zu sehen, der eine Abkehr von rein ökonomisch ausgerichteten Entwicklungsvorstel- lungen und eine Rückbesinnung auf die Region als integralen Lebensraum mit sich bringe. Die Schlagworte «Small is beautiful» und «Kleine Netze» beschreiben diese regionalpolitische Strategie prägnant.

Die eng verwandte Strategie der endogenen Regionalpolitik dagegen ist nach der Definition von ZIMMERMANN/NIJKAMP stärker ökonomisch ausgerichtet, wobei hier der Schwerpunkt der Analyse und die Ansatzpunkte der Regionalpolitik bei den endogenen Eigenschaften oder Potentialen der Regionen liegen. Diese Umorientierung sei vor allem durch die geringen Erfolge der traditionellen Regionalpolitik bedingt. Mit endogener Regionalpolitik sollen vor allem die intraregionalen Wirtschaftskreisläufe gestärkt, die regionale Wertschöpfung erhöht und eine selektive Abkoppelung vom überregionalen Markt erreicht werden.

Als eine Variante von endogener Regionalpolitik bezeichnet HAHNE $(1984,54)$ die «ökologische Regionalentwicklung», welche versucht, «auf der Grundlage der jeweils regional vorhandenen Ressourcen eine den ökologischen Bedingungen angepaßte Entwicklung einzuleiten, die von der Breite der Bevölkerung getragen wird. Im wesentlichen werden so die Ziele der sozialen, kulturellen, ökonomischen und ökologischen Entwicklung regional definiert und verfolgt.»

Die innovationsorientierte Regionalpolitik (vgl. z. B. BRUGGER 1983) ist ebenfalls als spezieller Ansatz endogener Strategien insoweit zu sehen, als diese Strategie das regionale Innovationspotential als wesentlichen Einflußfaktor regionaler Entwicklung in den Fördergebieten hervorstreicht. Es wird allerdings die Einbindung in den überregionalen Markt gesucht. Die Stellung der Region soll aber durch die Anwendung neuer Technologien und die Entwicklung und Produktion humankapitalintensiver Güter von hoher Qualität und mit hoher Wertschöpfung verbessert werden.

Die umweltbezogenen Folgen dieser neueren Strategien können mangels empirischer Basis nur hypothetisch beurteilt werden, zumal die drei ersten ohnehin in reiner Form noch nirgends praktiziert werden und einen hohen Utopiegehalt aufweisen. In bezug auf den Regionalismus und die endogene Regionalpolitik sind zumindest ZIMMERMANN/NIJKAMP (1986, 74) skeptisch: «Daß (diese Strategien) a priori und generell umweltfreundlicher ausfallen soll(en) als eine stärker „exogen” bestimmte, ist eher eine ideologische als eine rational begründbare These.» Werde die Umweltpolitik auf die regionale Entscheidungsebene delegiert, so sei zu befürchten, daß-u. a. wegen der Nichtbeachtung von Spillovers - tiefere Umweltstandards eine Verschlechterung der Umweltqualität herbeiführen würden.

Dagegen wird die innovationsorientierte Regionalpolitik positiver beurteilt, wobei sich die folgende 
Argumentation auf industrielle Problemregionen bezieht: «Zu einer positiven Einschätzung bezüglich der Umweltaspekte berechtigt die innovationsorientierte Regionalpolitik zweifellos: Wenn sich in peripheren und strukturschwachen Regionen eher alte Industrien mit alten Technologien befinden, dann kann innovationsorientierte Regionalpolitik zur Modernisierung der Industriestruktur und zu einer Absenkung der Emissionsintensität führen» (ZIMMERMANN/NIJKAMP 1986, 80).

\subsection{Umweltpolitik als Mittel der Regionalpolitik}

In 4.2 kam dem Umweltschutz der Status einer Randbedingung zu. Im vorliegenden Abschnitt stellen wir einige Ansätze vor, die noch einen Schritt weiter gehen und die Umweltpolitik als Mittel der Regionalpolitik einsetzen. In Kapitel 2.3 wurden die raumwirtschaftlichen Effekte von Umweltexternalitäten dargelegt. Negative externe Effekte wurden als eine Ursache für übermäßiges Agglomerationswachstum, positive Umweltexternalitäten, welche ihren Ursprung meist im ländlichen Raum haben, als eine Ursache für dessen Entwicklungsrückstand bezeichnet. Durch Internalisierung der negativen und positiven Umweltexternalitäten könnte somit - neben einer verbesserten Umweltallokation - ein regionaler Ausgleich bewirkt werden. Diese Strategie kann als «internalisierungsorientierte Regionalpolitik» bezeichnet werden. Den Begriff prägte R. L. FREY (1979b, 127f.), wobei er vor allem die negativen Ballungskosten im Infrastrukturbereich im Auge hatte.

Einen konkreten Ansatz internalisierungsorientierter Regionalpolitik nach dem Grundsatz der Internalisierung von Umweltexternalitäten stellt die von FRITSCH (1978 und 1980) vorgeschlagene Agglomerationsbesteuerung dar. Er schlug u. a. eine «Flächensteuer» in größeren Agglomerationen vor, die die Bodennutzer nach Maßgabe der beanspruchten Fläche zu bezahlen hätten, da eine solche Steuer seines Erachtens die verursachten Agglomerationskosten am besten widerspiegelt. Die Steuer ist sowohl als negativer Ballungsanreiz als auch als Finanzierungsquelle für regionalpolitische Fördermaßnahmen gedacht.

Der Autor des vorliegenden Beitrages bearbeitet gegenwärtig im Rahmen seiner Dissertation das Thema der internalisierungsorientierten Regionalpolitik nach dem Grundsatz der Internalisierung von Umweltexternalitäten auf umfassende Weise, d. h. unter Berücksichtigung negativer und positiver Umweltexternalitäten.

Einen ganz anderen Ansatz, Umweltpolitik als Mittel der Regionalpolitik einzusetzen, stellen Vorschläge dar, welche mit dem Begriff «nachfrageorientierte Wirtschaftspolitik des Staates» beschrieben werden können. Diese in der Schweiz wenig bekannten Konzepte schlagen vermehrte staatliche
Ausgaben in regionalpolitischen Problemregionen vor, wobei die Mittel gezielt in den Umweltsektor (Gewässerschutz usw.) zu lenken wären. Solche kombinierten Beschäftigungs-Umweltschutz-Programme werden vornehmlich von gewerkschaftlicher und sozialdemokratischer Seite vorgebracht (vgl. z. B. DEUTSCHER GEWERKSCHAFTSBUND 1988) und von liberaler Seite in erster Linie nach ordnungspolitischen Gesichtspunkten kritisiert.

\section{Die ökologische Dimension in der Raumplanung}

Abschließend wenden wir uns noch einem Teilbereich der Raumordnung zu, den wir bisher nur wenig beachtet haben: der Raumplanung. Der auftauchende Begriff der «ökologischen Planung» deutet an, daß auch hier Raumordnung und Umwelt vermehrt ineinandergreifen. Allerdings besitzt die Raumplanung definitionsgemäß einen Umweltbezug. Wenn Raumplanung die Aufgabe hat, Nutzungsansprüche an die knappe Ressource «Raum» zu ordnen und zu koordinieren, so ist sie automatisch auch «ökologisch orientiert», indem sie die für Natur und Gesellschaft lebenswichtigen Freiraumfunktionen (Landwirtschafts-, Erholungs-, Wald-, Naturschutzgebiete usw.) zu schützen versucht. Der Begriff der ökologischen Planung muß deshalb als Ausdruck dafür verstanden werden, daß ökologischen Aspekten bisher zu wenig Rechnung getragen wurde:

- Erstens ist der quantitative Schutz der Freiraumfunktionen insgesamt ungenügend, weil dominantere Bodennutzungsarten sich durchzusetzen vermögen. Im besonderen wird den für die Erhaltung der Funktionsfähigkeit des Naturhaushaltes wichtigen Schutzzonen zu wenig Gewicht beigemessen.

- Zweitens werden in qualitativer Hinsicht ökologische Aspekte zu wenig berücksichtigt, da die Freiraumfunktionen zu stark in bezug auf den Menschen interpretiert werden, und weil teilweise auch das notwendige ökologische Grundlagenwissen fehlt.

- Drittens wird die Koordinations- und Ordnungsfunktion der Raumplanung zu wenig auf das Umweltschutzziel ausgerichtet (räumliche Trennung konfliktreicher Aktivitäten usw.) (FÜRST 1986, 107f., SCHMID 1986).

Vor diesem Hintergrund können die Anliegen der ökologischen Raumplanung wie folgt umschrieben werden:

- Stärkere Berücksichtigung der Bedingungen und Erfordernisse des Naturhaushaltes sowohl bei der quantitativen Flächenausscheidung als auch bei der Nutzung der Flächen.

- Vermehrtes Ausschöpfen des Koordinations- und Steuerungspotentials der Raumplanung im Hinblick auf den Umweltschutz. 
Einen guten Einblick in das Konzept und in die Praxis der ökologischen Planung bieten REITH u. a. 1986 sowie die Sondernummer der DISP (Nr. 59/60) zur «Ökologie in der Raumplanung».

Die Berücksichtigung ökologischer Erfordernisse in der Raumplanung kann zu Konflikten mit regionalpolitischen Zielsetzungen führen. Verschiedene Autoren (z. B. HAMPICKE 1987) gehen davon aus, daß zur Sicherung der Funktionsfähigkeit des Naturhaushaltes die heute sehr intensive Landnutzung extensiviert werden müsse und insbesondere ein gewisser Anteil als nur mehr schwach genutzter ökologischer Ausgleichsraum auszuscheiden sei. Eine durch die Erfordernisse der Ökologie bedingte Raumstruktur nach dem Konzept der funktionsräumlichen Arbeitsteilung wirft die Frage auf, ob diejenigen Räume, denen ökologische Ausgleichsfunktionen zugewiesen werden, für die Nutzungs- und Entwicklungsbeschränkungen zu entschädigen wären. Da die durch die Extensivierung gesicherten ökologischen Funktionen des ländlichen Raumes gleichzeitig auch als gemeinwirtschaftliche Leistungen interpretiert werden können, wurde verschiedentlich eine Abgeltung durch die Allgemeinheit vorgeschlagen (vgl. z. B. HOLLENSTEIN/LOERTSCHER 1980, 50; BENKERT 1981, 196; BRÖSSE 1982, 98; R. L. FREY 1979b, 129). In der Schweiz stand die explizite Abgeltung dieser gemeinwirtschaftlichen Leistungen übrigens einmal ernsthaft zur Diskussion. Das Bundesgesetz über die Raumplanung vom 4. Oktober 1974, welches in der Volksabstimmung vom 13. Juni 1976 - knapp - verworfen wurde, sah in Artikel 45 einen «volkswirtschaftlichen Ausgleich zugunsten der Land- und Forstwirtschaft als Abgeltung für die Auflagen und Leistungen im Interesse der Raumplanung» vor, der aus der in Artikel 37 geregelten Mehrwertabschöpfung finanziert worden wäre.

\section{Schlußbemerkungen}

Versuchen wir eine Bilanz zu ziehen, so ist zunächst einmal festzustellen, daß die meisten Ansätze relativ neuen Datums sind. Meist weisen sie noch theoretisch-hypothetischen Charakter auf; praktische Erfahrungen oder empirische Untersuchungen bestehen erst vereinzelt. Ferner gilt, daß im Grenzbereich zwischen Raumordnung und Umwelt keine konsistente Forschungskonzeption vorliegt. Die Integrationsansätze sind vielmehr i.d. R. isolierte «Auswüchse» etablierter Forschungsbereiche wie etwa der Regionalökonomie, der Umweltökonomie oder der Raumplanung. Explizite Querbezüge zwischen den verschiedenen Integrationsansätzen bestehen kaum. Die markante Zunahme an Arbeiten im Grenzbereich zwischen Raumordnung und Umwelt deutet allerdings darauf hin, daß es sich dabei um ein Forschungsgebiet von breiterem Interesse handelt.

In diesem Beitrag wurde lediglich versucht, die verwirrende Vielfalt an Ansätzen und Arbeiten zu ordnen. Wir sind uns bewußt, daß möglicherweise dieser oder jener Aspekt verlorengegangen ist oder daß Gewichtung und Gliederung in gewissen Fällen auch anders hätten ausfallen können. Der knappe zur Verfügung stehende Raum erlaubte leider keine eingehenden Analysen der einzelnen Ansätze. Wir hoffen zumindest, denjenigen Personen, die an einer Stelle in diesem breiten Integrationsbereich tätig oder engagiert sind und von einer speziellen Sichtweise geprägt sind, andere Berührungspunkte zwischen Raumordnung und Umwelt in Erinnerung gerufen und die übrigen Leser für dieses zunehmend wichtiger werdende Forschungsgebiet interessiert zu haben.

\section{Literaturverzeichnis}

BAHRENBERG, G. (1983): Von der Anthropogeographie zur Regionalforschung - eine Zwischenbilanz. In: SEDLACEK P. (Hrsg.) 1983: Zur Situation der deutschen Geographie zehn Jahre nach Kiel. Osnabrücker Studien zur Geographie. Band 2, S. 59-68. Osnabrück.

BENKERT, W. (1981): Die raumwirtschaftliche Dimension der Umweltnutzung, Berlin.

BRÖSSE, U. (1982): Raumordnungspolitik, Berlin.

BRUGGER, E. A. (1983): Innovationsorientierte Regionalpolitik: Motive, Voraussetzungen und konzeptionelle Weiterentwicklung. In: DISP Nr. 72, S. 33-41.

BRUGGER, E. A. (1984): Endogene Entwicklung: Ein Konzept zwischen Utopie und Realität. In: Informationen zur Raumentwicklung 1/2 1984, S. 1-20.

BRUGGER, E. A. (1985): Regionalwirtschaftliche Entwicklung, Bern.

BRUGGER, E. A./FREY, R. L. (1985): Regionalpolitik Schweiz, Bern.

Bundesgesetz über die Raumplanung vom 4. Oktober 1974.

Deutscher Gewerkschaftsbund (1988): Strukturprogramm Küste, Hamburg.

DISP (1980): Ökologie in der Raumplanung, Nr. 59/60, ORLInstitut der ETH Zürich.

FREY, B. S. (1985): Umweltökonomie, Göttingen.

FREY, R. L. (1979 a): Interregional welfare comparisons and environmental policy. In: Siebert u. a. 1979, S. 97-108.

FREY, R. L. (1979 b): Infrastruktur als Mittel der Regionalpolitik, Bern.

FREY, R. L. (1985): Regionalpolitik - eine Evaluation, Bern.

FREY, R. L./GYSIN, C. H./LEU, R. E./SCHMASSMANN, N. (1985): Energie, Umweltschäden und Umweltschutz in der Schweiz, Grüsch.

FRITSCH, M. (1978): "Agglomerationsbesteuerung" als Instrument der Regionalpolitik in der Bundesrepublik Deutschland? In: Informationen zur Raumentwicklung 4/1978. 
FRITSCH, M. (1980): Begründung und Ausgestaltung einer "Agglomerationsbesteuerung", IIM-Discussion Paper 80-71, Berlin.

FÜRST, D. (1986): Die Problematik einer ökologisch orientierten Raumplanung. In: Fürst u. a. 1986, S. 103-213.

FÜRST, D./NIJKAMP, P./ZIMMERMANN, K. (1986): UmweltRaum-Politik, Berlin.

HAHNE, U. (1984): Ökologische Regionalentwicklung. In: Informationen zur Raumentwicklung 1/2 1984, S. 53-62.

HAMPICKE, U. (1987): Ökologische Vorgaben für die Agrarökonomie, IIUG rep 87-10. Berlin.

HOLLENSTEIN, H./LOERTSCHER, R. (1980): Die Strukturund Regionalpolitik des Bundes, Diessenhofen.

KRIPPENDORF, J. (1984): Das Kapital des Tourismus in Gefahr. In: BRUGGER, E. A., FURRER, G., MESSERLI, P.: Umbruch im Berggebiet, Bern, S. 601-630.

KRIPPENDORF, J./KRAMER, B./MÜLLER, H.-R. (1987): Freizeit und Tourismus, Bern.

LENDI, M./ELSASSER, H. (1985): Raumplanung in der Schweiz, Zürich.
LESER, H. (1980): Geographie, Braunschweig.

REITH, W. J./LENDI, M./SCHMID, W. A. (1986): Ökologische Planung im Grenzraum, Wien.

LLOYD, P. E./DICKEN, P. (1977): Location in space, London. RICHARDSON, H. W. (1978): Urban economics, Hinsdale. SCHÄTZL, L. (1981): Wirtschaftsgeographie 1, Paderborn. SCHMID, W. A. (1986): Ökologische Planung - eine Aufgabe der Raumplanung. In: REITH u. a., S. 367-384.

SIEBERT, H. (1978): Ökonomische Theorie der Umwelt, Tübingen.

SIEBERT, H. (1979): The regional dimensions of environmental policy. In: SIEBERT u. a., S. 1-12.

SIEBERT, H.MALTER, I./ZIMMERMANN, K. (1979): Regional environmental policy, New York.

ZIMMERMANN, K. (1985): Umweltpolitik und Verteilung, Berlin.

ZIMMERMANN, K./NIJKAMP, P. (1986): Umweltschutz und regionale Entwicklungspolitik - Konzepte, Inkonsistenzen und integrative Ansätze. In: FÜRST u. a., S. 20-101. 